\title{
Zur Beziehung zwischen Literatur und Recht in der deutschsprachigen Gegenwartsliteratur
}

\author{
[The Relationship between Law and Literature in Contemporary German Literature]
}

Sonja Arnold ${ }^{1}$

\begin{abstract}
This paper examines the relationship between law and literature in contemporary German literature. Current trends of this relationship will be discussed, starting from the long tradition of the so-called Dichterjuristen (lawyers who are also writers), such as Goethe, Kleist and Kafka. Firstly, theoretical backgrounds of law in literature will be presented, after that specific topics, genres and aesthetic particularities of the texts in question will be identified. The contribution analyzes novels by the German authors Juli Zeh and Ferdinand von Schirach and their adaptions for cinema and TV (the TV series Crime for the German TV channel ZDF based on Ferdinand von Schirach's collection of short stories of the same title, and the miniseries $A$ Menina sem Qualidades, an adaptation of Juli Zeh's novel Spieltrieb for MTV Brazil).
\end{abstract}

Keywords: Contemporary literature; Law and Literature; Juli Zeh; Ferdinand von Schirach

Zusammenfassung: Dieser Beitrag untersucht die Beziehung zwischen den Diskursen Literatur und Recht in der deutschsprachigen Gegenwartsliteratur. Ausgehend von der langen Tradition der sogenannten Dichterjuristen (Goethe, Kleist, Kafka) werden aktuelle Tendenzen dieser Beziehung in den Blick genommen. Nach theoretischen Überlegungen zum Teilaspekt law in literature (Recht in der Literatur) erfolgt die Bestimmung von spezifischen Themen, Genres und ästhetischen Besonderheiten der in Frage stehenden Texte. Als Textgrundlage dienen Romane von Juli Zeh und Ferdinand von Schirach. In dieser Analyse werden auch die Adaptionen für Kino und TV einbezogen (die Serie Verbrechen für den TV-Sender ZDF, die auf Ferdinand von Schirachs gleichnamiger Kurzgeschichtensammlung beruht, und die Miniserie A Menina sem Qualidades, eine Adaption von Juli Zehs Spieltrieb für MTV Brasilien).

Stichwörter: Gegenwartsliteratur; Recht und Literatur; Juli Zeh; Ferdinand von Schirach

Resumo: A contribuição visa a investigar a relação entre os discursos do direito e da literatura na literatura contemporânea de língua alemã. Baseado na longa tradição dos Dichterjuristen (advogados-escritores) como Goethe, Kleist e Kafka, pretende-se discutir as atuais tendências no estudo de tais relações. Começa-se com reflexões teóricas sobre law in literature (direito na literatura), a fim de, então, identificar temas, gêneros e uma estética/narrativa específica dos textos contemporâneos em questão. Como objeto de análise, discutem-se os romances de Juli Zeh e Ferdinand von Schirach. Serão também consideradas as respectivas adaptações para cinema ou televisão (a série Verbrechen (Crimes) no programa alemão ZDF, e A menina sem qualidades, adaptação do romance Spieltrieb de Juli Zeh para o MTV Brasil).

Palavras-chave: Literatura contemporânea Direito e Literatura; Juli Zeh; Ferdinand von Schirach

\footnotetext{
${ }^{1}$ DAAD-Lektorin an der Universidade Federal do Rio Grande do Sul. E-Mail:

daad_porto_alegre@daad.org.br
}

Pandaemonium, São Paulo, v. 17, n. 23, Jun./2014, p. 1-25 
Arnold, S. - Zur Beziehung zwischen Literatur und Recht

\section{Einführung}

Schreibende Juristen, die in fiktionalen Texten juristische Problemfelder thematisiert, transformiert und unter fiktiven Rahmenbedingungen durchgespielt haben, gibt es in der Kulturgeschichte seit Jahrhunderten. Angefangen mit Goethe, über Heinrich von Kleist bis Franz Kafka lassen sich spektakuläre Fälle, Verbrecherbiographien, Psychologisierungen und rechtsphilosophische Fragestellungen nachzeichnen, deren Verhandlung im 18. Jahrhundert in Schillers Verbrecher aus verlorener Ehre genauso aktuell war wie in den berühmten Kriminalgeschichten Friedrich Dürrenmatts Mitte des 20. Jahrhunderts. Ebenso vielfältig sind mittlerweile die theoretischen Untersuchungen zu den Begegnungsfeldern zwischen den beiden Disziplinen Literatur und Recht. Das sich in den 1970er-Jahren in den USA zunehmend systematisch formierende Forschungsfeld Law and Literature Movement ${ }^{2}$ unterscheidet hierbei zunächst grob zwischen dem Recht in der Literatur ${ }^{3}$ (Darstellung von Verbrechen, Richtern, Anwälten oder Gerichtsverfahren in fiktionalen Texten), dem Recht als Literatur ${ }^{4}$ (narrative Struktur von juristischen Texten, literaturtheoretische Konzepte, die in den Rechtswissenschaften zur Anwendung kommen) und dem Recht der Literatur (hier stehen vor allem Fragen des geistigen Eigentums und Urheberrechts im Vordergrund). ${ }^{5}$ Innerhalb des letzten Feldes wurden insbesondere in den letzten Jahren einige öffentlichkeitswirksame Gerichtsverfahren geführt, in denen die Darstellung realer Personen in literarischen Texten und der Fiktionalitätsstaus der Werke im Mittelpunkt standen. ${ }^{6}$

\footnotetext{
${ }^{2}$ Die Bewegung wird oftmals mit dem Erscheinen von James Boyd Whites The Legal Imagination angesetzt, wenngleich sie auch in John Wigmore oder Benjamin Cardozo um die Jahrhundertwende vom 19. zum 20. Jahrhundert frühere Vorläufer hatte (vgl. DuONG 2005: 5).

${ }^{3}$ Law in Literature: Diese Einteilung geht auf eine bereits 1908 von John Wigmore erstellte Liste literarischer Werke zurück, wonach jeder Jurist zum besseren Rechtsverständnis bestimmte literarische Werke gelesen haben sollte.

${ }_{5}^{4}$ Als Gründervater gilt hier Benjamin N. Cardozo.

${ }^{5}$ Jüngst wurden diese Kategorien durch zahlreiche Subkategorien erweitert. Vgl. hierzu die von Arnaldo SAMPAIO DE MORAES GODOY (2008: 23-39) entwickelten Kategorien Direito $n a$ literatura (Recht in der Literatur), Direito como literatura (Recht als Literatur), direito da literatura (Recht der Literatur), literatura como veículo do direito (Literatur als Wegbereiter für das Recht).

${ }^{6}$ In Deutschland betrafen einige dieser Fälle in den letzten Jahren: Maxim Billers Esra, Birgit Kempkers Als ich das erste Mal mit einem Jungen im Bett lag und Alban Nicolai Herbsts Meere. In Brasilien richtete sich jüngst der Protest einiger Gegenwartsautoren gegen die Einschränkung der Pressefreiheit, der sich an den Kontroversen um eine Biographie über Roberto Carlos und einem anschließenden Gerichtsverfahren entzündet hatte: http://www1.folha.uol.com.br/ilustrada/2013/09/1338851-na-bienalruy-castro-apresenta-manifesto-contra-censura-a-biografias.shtml (19/05/2014).
} 
Arnold, S. - Zur Beziehung zwischen Literatur und Recht

Ein Blick auf die Vielzahl von Publikationen zu den Wechselbeziehungen zwischen den Diskursen Literatur und Recht zeigt, dass ein Großteil sich vornehmlich mit den Implikationen einer Sichtweise von Recht als Literatur befasst. ${ }^{7}$ Diese meist von Juristen, insbesondere als Zweig verschiedener US-amerikanischer Law Schools, betriebene Forschung beschäftigt sich hauptsächlich mit der narrativen Analyse juristischer Texte mithilfe literaturwissenschaftlicher Methodik sowie mit der Applikation literaturtheoretischer Konzepte auf juristische Texte (vgl. BARON/EPSTEIN 1997). Diesen relativ systematischen Überlegungen steht auf der anderen Seite (Recht in der Literatur) eine mittlerweile nahezu unüberschaubare Anzahl an Einzelanalysen literarischer Rechtsfälle gegenüber, die indes nicht immer auf theoretische Überlegungen zum Verhältnis von Literatur und Recht gegründet sind. ${ }^{8}$ Die genannten Unterscheidungsformen sind beim Überblicken der zahlreichen Wechselbeziehungen zwischen Recht und Literatur grundsätzlich hilfreich, gehen aber mit einigen grundlegenden Problemen einher. Zunächst scheinen durch die Unterteilung in die genannten Kategorien thematische und ästhetische Ausgestaltung strikt voneinander getrennt. So dominieren bei der Analyse juristischer Themen in der Literatur meist stoffliche Fragestellungen, während die ästhetische Analyse, die die Verwendung von Metaphern, rhetorischen Figuren sowie die textuelle Anordnung untersucht, tendenziell auf die Analyse juristischer Texte mithilfe literaturwissenschaftlicher Kategorien beschränkt bleibt. Des Weiteren werden durch die Wahl eines enger gefassten Textbegriffs (literature), der sich auf (meist zur Höhenkammliteratur zählende) schriftliche Zeugnisse bezieht, andere mediale Vermittlungsformen sowie intermediale Transformationsprozesse (z. B. vom Roman zum Film) ausgeblendet. Schließlich wird durch die Präposition in im Ausdruck Recht in der Literatur der Anschein erweckt, dass es sich um eine unidirektionale Beziehung handelt, Literatur mithin rechtliche Fragestellungen lediglich darstellt, jedoch keine Rückwirkungen auf andere Diskurse hat. Anliegen des vorliegenden Beitrags ist es daher, nach spezifischen

\footnotetext{
${ }^{7}$ Vgl. stellvertretend: BARON/EPSTEIN (1997: 141-187). Vgl. auch die Bibliographie des European Network for Law and Literature:

http://www.esl.eur.nl/fileadmin/ASSETS/frg/arw/rechtstheorie/Law__Literature_Bibliography_Greta_O lson__Jeanne_Gaakeer.pdf (27/01/2014).

${ }^{8}$ Einige Forschungsprojekte und Publikationen der letzten Jahre bilden hier jedoch einen sehr fruchtbaren Ansatz, indem sie nach den kulturellen (vgl. OLSON 2010 und OLSON 2013), historischen (vgl. FLUDERNIK/OLSON 2004, MÖLK 1996, SCHÖNERT 1991) und systematischen Implikationen (WEITIN 2010, MÜLLER-DIETZ 1990, LÜDERSSEN 1991) sowie nach den dynamischen Wechselbeziehungen zwischen den Systemen Recht und Literatur fragen.
} 
Arnold, S. - Zur Beziehung zwischen Literatur und Recht

Darstellungsformen rechtlicher Problemfelder in der Literatur, ihren spezifischen Funktionen und den Wechselwirkungen mit nichtliterarischen Diskursen zu fragen.

In der gegenwärtigen Literatur lässt sich mit Blick auf die deutschsprachige Literatur geradezu ein Boom in Produktion und vor allem auch Rezeption der Texte von sogenannten Dichterjuristen feststellen. Hierzu gehören Rolf Henrich, Martin Mosebach, Georg M. Oswald, Ferdinand von Schirach, Bernhard Schlink und Juli Zeh, wobei hier bei weitem kein Anspruch auf Vollständigkeit erhoben wird. Von der Mehrheit der genannten Autoren liegen zudem Übersetzungen ins brasilianische Portugiesisch vor und sie sind insbesondere auch durch ihre Adaptionen für Kino und TV populär geworden. ${ }^{9}$ Anhand von zwei Beispielanalysen gegenwärtiger deutschsprachiger Romane, die juristische Problemfelder thematisieren und darstellen, werden im Folgenden ästhetische Besonderheiten, ihre Funktionen sowie die Wechselwirkungen mit nichtliterarischen Diskursen herausgearbeitet. Dabei kann auf von der Forschung bereits postulierte Spezifika der literarischen Darstellung von juristischen Problemfeldern wie die Neuverknüpfung und das Hinzufügen einzelner Elemente im literarischen Text (vgl. SCHÖNERT 1991: 15), die Infragestellung allgemeiner Gerechtigkeitserwartungen (vgl. MüLLER-DIETZ 1990: 20), die Funktion der „Literatur als Unfallschauplatz“ (WEITIN 2010: 36) sowie ihre Fähigkeit zum Entwurf möglicher Welten (vgl. LÜDERSSEN 1991: 12 f.) zurückgegriffen werden.

Dies geschieht am Beispiel von zwei deutschen Autoren, die (a) sowohl Juristen als auch Schriftsteller sind, (b) in Deutschland und international durch ihre Werke und intermediale Adaptionen derselben bekannt geworden sind und die (c) im Sinne einer littérature engagée über $\mathrm{ihr}$ literarisches Schaffen hinaus an gesellschaftlichen Diskussionen (mit juristischem Hintergrund) partizipieren. So können theoretische, nicht-fiktionale Äußerungen der Autoren zu der fiktionalen Verarbeitung in Beziehung gesetzt und im kontrastiven Vergleich nach Spezifika der literarischen Produktion gefragt werden. Ziel des Beitrags ist eine Herausarbeitung der spezifischen Funktionen und ästhetischen Besonderheiten literarischer Rechtsnarrative in der gegenwärtigen deutschsprachigen Literatur. Hierzu werden Juli ZEHS (2004) Roman Spieltrieb, unter Berücksichtigung seiner Übersetzung ins brasilianische Portugiesisch

\footnotetext{
${ }^{9}$ Beispielsweise die Verfilmung von Bernhard Schlinks Roman Der Vorleser, die Adaption von Ferdinand von Schirachs Erzählband Verbrechen als TV-Serie für das ZDF und der daraus entstammenden Erzählung Glück als Spielfilm durch Doris Dörrie sowie die Adaption der Übersetzung von Juli Zehs Roman Spieltrieb als Miniserie für MTV Brasilien unter dem Titel A Menina sem Qualidades.
} 
Arnold, S. - Zur Beziehung zwischen Literatur und Recht

durch Marcelo Backes (ZEH 2009) und seiner Adaption als Miniserie für MTV Brasil ${ }^{10}$ sowie Ferdinand von SCHIRACHS (2009) Kurzgeschichtensammlung Verbrechen und ihre Adaption als Serie für das ZDF (2013) sowie sein jüngster Roman Tabu (SCHIRACH 2013) untersucht. Unter Berücksichtigung folgender Punkte wird beleuchtet, wie in den Romanen und TV-Adaptionen die Verarbeitung von genuin juristischen Stoffen mit literarischen Mitteln durchgeführt wird und in welchem Spannungsverhältnis sie zum juristischen Diskurs stehen:

1) Rechtsphilosophische Überlegungen (Verhältnis von Recht und Gerechtigkeit, Verhältnis von einzelnem und System) und ihre Konsequenzen für die literarische Form;

2) Das literarische Durchspielen eines konkreten Falls und seiner Konsequenzen anhand des spieltheoretischen Konzepts Gefangenendilemma;

3) Die literarische Inszenierung einer Gerichtsverhandlung und ihre ästhetischen Besonderheiten;

4) Die Rolle von Gattung und Erzählinstanz (Moderne Pitavalgeschichten);

5) Fiktionalisierung von politischen Manifesten.

\section{Juli Zeh Spieltrieb (2004)}

\subsection{Zur Kontinuität des essayistischen Romans: Juli Zehs Spieltrieb und Robert Musils Mann ohne Eigenschaften}

Spieltrieb (2004) ist Juli Zehs zweiter Roman. Die 1974 geborene Autorin und promovierte Juristin hat jüngst durch einen Aufruf von internationaler Tragweite Aufsehen erregt. Die Demokratie verteidigen im digitalen Zeitalter heißt der Appell, der am 10.12.2013 unter anderem in der FAZ veröffentlicht und von über tausend Schriftstellern aus der ganzen Welt unterzeichnet wurde. ${ }^{11}$ Der Aufruf versteht sich als Protest gegen eine systematische Überwachung im Internet - eine Diskussion, an der Juli Zeh bereits seit längerem beteiligt ist und die durch die jüngsten Skandale um den

\footnotetext{
${ }^{10} \mathrm{http} / / \mathrm{mtv}$.uol.com.br/programas/ameninasemqualidades/blog/a-menina-sem-qualidades-estreia-namtv-brasil-no-dia-27-de-maio (27/01/2014).

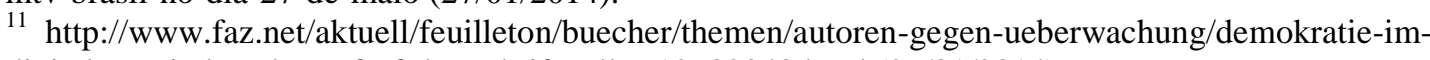
digitalen-zeitalter-der-aufruf-der-schriftsteller-12702040.html (27/01/2014).
} 
Arnold, S. - Zur Beziehung zwischen Literatur und Recht

US-amerikanischen Geheimdienst NSA neue Brisanz gewonnen hat. Ihre regelmäßigen TV-Auftritte in politischen Sendungen, das gemeinsam mit Ilja Trojanow verfasste Manifest Angriff auf die Freiheit. Sicherheitswahn, Überwachungsstaat und der Abbau bürgerlicher Rechte (2009) sowie die Zukunftsdystopie Corpus Delicti (2009) markieren des Weiteren ihre intensive Auseinandersetzung und Einflussnahme auf aktuelle politische und juristische Diskussionen. Aufgrund der Vielzahl von theoretischen Äußerungen, die im Fall von Juli Zeh vorliegen (vgl. ZEH/OsWALD 2011) und teilweise in identischer Form in ihren Romanen auftauchen, bietet sich ein Vergleich von per se nichtliterarischen Diskursen mit ihrer literarischen Verarbeitung besonders an.

"Wenn das alles ein Spiel ist, sind wir verloren. Wenn nicht - erst recht" (ZEH 2004: 10). So endet der einleitende Teil des 82 Kapitel umfassenden Romans Spieltrieb (2004) von Juli Zeh. Die vierzehnjährige Ada, Schülerin des elitären Ernst-BlochGymnasiums, ist ihren Mitschülern intellektuell weit überlegen, was sie unter anderem durch die Lektüre von Robert Musils Roman Der Mann ohne Eigenschaften demonstriert. In Alev, einem neuen, drei Jahre älteren Mitschüler, findet sie ihr intellektuelles Pendant. Die beiden bezeichnen sich selbst als „Urenkel der Nihilisten“ (ZEH 2004: 7) und versuchen im weiteren Verlauf des Romans, die Grenzen im Umgang mit ihren Mitmenschen auszuloten und zu überwinden. Angeregt durch die Lektüre von Robert Axelrods The Evolution of Cooperation (1984) beschließen die beiden, ein spieltheoretisches Szenario, das so genannte Gefangenendilemma, auf die Realität anzuwenden. Als Spielfiguren dienen ihnen hierbei Lehrer und Mitschüler; das Spiel verliert dabei seinen hypothetischen Charakter und bestimmt zunehmend das Zusammenleben von Schülern und Lehrern. Einem ersten Todesopfer in Form des Geschichtslehrers Höfi, der sich vom Dach der Schule in den Tod stürzt, folgt die Erpressung des Deutsch- und Sportlehrers Smutek. Dieser wird auf Alevs Anweisung von Ada verführt und mithilfe einer Videoaufzeichnung, die passwortgeschützt auf der schulinternen Internetplattform steht, zur wöchentlichen Wiederholung im Beisein Alevs gezwungen. Das Spiel gerät jedoch zunehmend außer Kontrolle, die Teilnehmer agieren nicht wie im spieltheoretischen Modell vorgesehen und es endet schließlich mit einem Akt purer Gewalt und Brutalität. Im anschließenden Gerichtsverfahren steht die kalte Sophie, eine erfahrene Richterin, ratlos den Aussagen der Beteiligten gegenüber, 
Arnold, S. - Zur Beziehung zwischen Literatur und Recht

während die Anwendung des bestehenden Rechts auf den vorliegenden Fall aufgrund der fehlenden Rückbindung an moralische Grundlagen nahezu unmöglich erscheint.

Diese Rahmenhandlung dient den Figuren und dem allwissenden Erzähler als Anlass $\mathrm{zu}$ ausführlichen, die Handlung unterbrechenden Reflexionen ${ }^{12}$ über Willensfreiheit und Entscheidungsgrundlagen (,Es gibt kein Für und Wider“, sagt, Ada, „keine Gründe für rechts oder links. Die menschliche Entscheidung ist nichts weiter als ein vortrefflich einstudiertes Spiel“, ZEH 2004: 192), Möglichkeiten der Wahrheitsfindung (,Wir werden die Wahrheit nicht erfahren. Der Punkt ist, dass die Wahrheit keine Rolle spielt.“ ZEH 2004: 241,) sowie über das Verhältnis von Recht und Gerechtigkeit („das Recht sei ein Raum, den die Gerechtigkeit niemals betrete“, ZEH 2004: 518). Die Reflexionen erfolgen entweder in der Figurenrede oder als längere, die eigentliche Handlung unterbrechende Reflexionen des Erzählers und stellen häufig eine Verbindung zu rechtlichen Fragestellungen her. Im Interview äußert Juli Zeh über den juristischen Stoff im Roman:

Spieltrieb, auf seinen Juragehalt befragt, beschäftigt sich mit der kleinstmöglichen Systemform, die es gibt, nämlich im Grunde mit dem Zusammenleben von zwei bis drei Personen und dem Versuch, innerhalb dieses winzig kleinen Gesellschaftsmodells Regeln zu setzen, die dann natürlich keine Rechtsnormen, sondern allenfalls Spielregeln sein können. Denn diese kleinen, in sich geschlossenen Systeme, die sich dennoch nach bestimmten Normen verhalten, sind im Grunde schon fast per definitionem Spiele. Und so behauptet das Buch, daß sozusagen der Verlust des Glaubens an ein übergeordnetes Rechtssystem bei den Einzelnen, bei den Individuen eine Art Spielverhalten erzeugt (WEBER 2007: 190).

Diese thematische Basis, die grundsätzliche Überlegungen zur Funktionsweise des Rechtssystems enthält, wird im Roman als Spiel modelliert, sie wird explizit von Figuren und Erzähler thematisiert und ist, so die These dieses Beitrags, auch mit verschiedenen ästhetischen Konsequenzen, die Bauform der Romans betreffend, verbunden. Als ästhetische Besonderheit ließen sich zunächst die essayistischreflektierenden Passagen bestimmen, die das Genre des Romans dem Essayismus annähern und damit gattungsgeschichtlich als Gedächtnis der Literatur (vgl. ERLL/NüNNING 2005: 2) an Romane wie Robert Musils Mann ohne Eigenschaften oder Thomas Manns Zauberberg erinnern. Auf beide Romane wird in Zehs Spieltrieb

\footnotetext{
${ }^{12}$ Wie der Übersetzer Marcelo Backes anmerkt, wird hierbei nicht immer deutlich zwischen den Gedanken einzelner Figuren und denjenigen der Erzählerin unterschieden, d.h. einige Gedanken der Figuren tauchen identisch in den Reflexionen der Erzählerin wieder auf (vgl. ZEH 2009: 533).
}

Pandaemonium, São Paulo, v. 17, n. 23, Jun./2014, p. $1-25$ 
Arnold, S. - Zur Beziehung zwischen Literatur und Recht

explizit Bezug genommen. Die Referenzen auf den Zauberberg lassen sich vornehmlich im Erwähnen der beiden zentralen Figuren Settebrini und Castorp (vgl. ZEH 2009: 534f) identifizieren, die Musil-Referenz zeigt sich in einer Vielzahl von Parallelen zum 74 Jahre vorher erschienenen Roman Der Mann ohne Eigenschaften. Die Struktur des Romans besteht, ähnlich wie bei Musil, aus kurzen Kapiteln, die in ihren Überschriften nahezu Epigrammen ähneln. Im Erzähltext wechseln Figurenrede mit beschreibenden Passagen sowie reflektierenden Passagen des Erzählers. In die erzählte Welt werden konkrete Musilsche Begrifflichkeiten wie „Aggregatzustand“ (ZEH 2004: 159), ${ }^{13}$ und "Versuchsanordnung" (z. B. ZEH 2004: 125, 319) integriert sowie detaillierte Wetterbeschreibungen, die an die berühmte Anfangspassage des Manns ohne Eigenschaften erinnern. Die Musil-Referenz wird zudem durch die gemeinsame Lektüre des Manns ohne Eigenschaften der Schulklasse explizit in den Roman integriert (in der MTV-Serie sieht der Zuschauer die Hauptfigur Ana den Roman in der Badewanne lesen, während die gemeinsame Lektüre der Schulklasse durch Julio Cortázars Rayuela ersetzt wird). Interessant hierbei sind auch die Titel der Übersetzungen in französische und portugiesische Sprache: La fille sans qualités und A menina sem qualidades ${ }^{14}$ (wörtlich: das Mädchen ohne Eigenschaften), die die Musil-Referenz bereits in den Titel aufnehmen. Die Aufnahme rechtsphilosophischer Fragestellungen erschöpft sich dabei nicht in einer thematischen Reminiszenz, sondern schlägt sich strukturell in der Gestaltung des Romans nieder, der in Form eines Gedächtnisses der Literatur gattungsgeschichtlich an die Tradition des essayistischen Romans anschließt und sie für rechtsphilosophische Überlegungen nutzt. Dabei wird an eine Gattung erinnert, deren genuine Eigenschaft in einem epistemologischen Verfahren, das auf subjektiver Erfahrung gründet (vgl. SCHLAFFER 1997: 522), beruht, und die es in diesem Fall vermag, objektive Überlegungen zur Grundlage des Rechtssystems mit hypothetischen, spieltheoretischen Szenarien zu verbinden. Innerhalb dieser Form lassen sich immer wieder Reflexionen zum Verhältnis von Wirklichkeit und Möglichkeit nachzeichnen, die ebenso an Musils Termini Wirklichkeitssinn und Möglichkeitssinn (MusIL 2005 [1930]: 16) erinnern:

\footnotetext{
${ }^{13}$ Vgl. dazu auch Juli Zehs Roman Schilf: "wechselte die Wirklichkeit ihren Aggregatzustand von gasförmig zu fest" (ZEH 2009: 16).

${ }^{14}$ Dies ist nach Angaben des Übersetzers unter anderem der doppelten Bedeutung von „Spiel“ im Deutschen geschuldet, die sowohl das Portugiesische ,jogo“ als auch „brincadeira“ enthält. Der Titel wurde aufgrund einer Rezension in der FAZ mit dem Titel „Mädchen ohne Eigenschaften: Juli Zehs zweiter Roman" und in Anlehnung an die französische Übersetzung gewählt und erinnert an die MusilReferenz bereits im Titel (vgl. ZEH 2009: 540f).
} 
Arnold, S. - Zur Beziehung zwischen Literatur und Recht

Wenn der Mensch versucht, sich etwas vorzustellen, wird er notwendig irren. Vorstellung und Wirklichkeit sind nur in Ansätzen aufeinander bezogen, und der Glaube, Erstere müsse sich ständig mit letzterer befassen, beruht auf der Tatsache, dass jene unerträgliche Gleichung, die wir unser Ich nennen, genau am Schnittpunkt beider Koordinaten liegt (ZEH 2004: 379).

An dieser Stelle setzt der Spielbegriff an. Spiele ermöglichen einerseits als regelgeleitete Systeme theoretische Voraussagen über die eintretenden Züge der beteiligten Spieler. Andererseits erlaubt die Sichtweise eines Spiels als Probehandeln gerade das Durchdenken einer Vielzahl von möglichen Zügen und Alternativen. Künstlerische und literarische Realisierungen von Spielen können dabei als Simulationsräume fungieren (vgl. NeuHAus 2009: 388), die hier eine Verbindung zu rechtlichen Fragestellungen aufweisen und ihr gattungsspezifisches Pendant im experimentellen Charakter des Essayismus (SCHLAFFER 1997: 525) erhalten. Dass die Vorstellbarkeit von verschiedenen Szenarien und ihrer Kombinationsmöglichkeiten in der Realität aber nicht immer zu genauen Aussagen führt, zeigt sich im Scheitern des Gefangenendilemmas im Roman. Wirklichkeit und Möglichkeit, die in Zehs Roman im Begriff des Spiels zusammentreffen und in der Form des essayistischen Romans die subjektive und experimentelle Komponente ausführlich zu kommentieren vermögen, liegen als Grundsatzpaar auch dem Rechtssystem zu Grunde, insbesondere dann, wenn es sich um Recht in der Tradition des code law systems handelt, d.h. um ein Rechtssystem, dessen Anspruch es ist, eine gültige Regel für alle möglichen Szenarien zu Grunde zu legen. ${ }^{15}$ Die Ausarbeitung möglicher Szenarien, die auf wirklichen Gesetzen basieren, kann damit auch als Reflexion über die Funktionsweise des Rechts gelesen werden. Die literarische Form bietet mithin ein „Experimentierfeld“ (SCHÄRF 1999: 10) ${ }^{16}$ für verschiedene rechtsphilosophische Reflexionen und das Ausarbeiten verschiedener möglicher Szenarien .

\footnotetext{
${ }^{15}$ Dies gilt zumindest für diejenigen Rechtssysteme, die als code law systems fungieren, d.h. auf alle möglichen Fälle anwendbare Formulierungen enthalten. Im Falle des US-amerikanischen Rechtssystems, das ein case law system, also auf einzelnen konkreten Fällen beruhendes Rechtssystem darstellt, hat dies weniger Relevanz. (Vgl. BRUGGER / SARLET 2008).

${ }^{16}$ Vgl. hierzu auch Juli Zehs Überlegungen zur Literatur als Spielfeld: „Wenn die Literatur ein Spielfeld und eine Tanzfläche für die Kunst der Erinnerung ist, eine lustvolle Technik zur Erzeugung von Vergangenheit, dann ist Politik Spielfeld und Tanzfläche für die Kunst der Zukunftsgestaltung, eine sinnvolle Methode zum Umgang mit Wünschen, Hoffnungen und Visionen.“ (ZEH/OswALD 2011: 91).
} 
Arnold, S. - Zur Beziehung zwischen Literatur und Recht

\subsection{Ein spieltheoretisches Szenario: Das Gefangenendilemma und Robert Axelrods The Evolution of Cooperation}

Das bereits mehrfach erwähnte Gefangenendilemma stammt ursprünglich aus der Spieltheorie und hat in mehreren Disziplinen Anwendung gefunden, insbesondere in der Wirtschaftswissenschaft, wenn es darum geht, Verhaltensweisen einzelner Akteure vorauszusagen. Genau an diesem Punkt treffen sich auch Recht und Wirtschaftswissenschaften und haben den fruchtbaren Zweig der Law and EconomicsBewegung ${ }^{17}$ hervorgebracht. In Zehs Roman wird besagtes Dilemma mit seinen verschiedenen Kooperationsmöglichkeiten entfaltet. ${ }^{18}$ Alev fungiert hierbei als Spielleiter: er gibt die Instruktionen, verteilt Belohnungen und Bestrafungen und setzt die Regeln fest. Zur Einführung befiehlt er Ada, den einleitenden Teil von Robert Axelrods The Evolution of Cooperation zu lesen (vgl. ZEH 2004: 191). Dieses Standardwerk der Spieltheorie stellt sich die Frage, wie Kooperation unter Spielern entstehen kann und welche Strategien sie dafür wählen müssen. Dazu nimmt Axelrod auch auf das Gefangenendilemma Bezug. In Anlehnung daran beschreibt Alev das Dilemma folgendermaßen:

Zwei Angeklagte werden einzeln dem Gericht vorgeführt. Jedem von ihnen schlägt der Richter einen Deal vor: Wenn du gestehst und deinen Kumpel verpfeifst, bleibst du straffrei und der andere kriegt fünf Jahre Knast. Schweigt ihr beide, reichen die Indizien für jeweils zwei Jahre. Gesteht ihr beide, bekommt jeder vier Jahre. Sie haben keine Möglichkeit, sich abzusprechen (ZEH 2004: 278 f.).

Das entworfene Schema gleicht in der Tat demjenigen, das Axelrod in der Einleitung seiner Evolution of Cooperation beschreibt. Formal ließe es sich folgendermaßen beschreiben: ${ }^{19}$

\footnotetext{
${ }^{17}$ Diese Bewegung entwickelte sich seit den frühen 1970er Jahren in den USA und hängt auch eng mit der Frage zusammen, ob die Rechtswissenschaft eher den Geistes- oder den Naturwissenschaften zugerechnet wird (vgl. BALKIN 2006: 160).

${ }^{18}$ Vgl. zu den folgenden Ausführungen ARNOLD 2011.

${ }^{19}$ Schema modifiziert nach AXELROD (2005 [1984]: 8). Die Spieler können sich in diesem Schema entweder für Kooperation oder Defektion (Nicht-Kooperation) entscheiden. Im ersten Quadranten kooperieren beide, indem sie schweigen und erhalten jeweils nur 2 Jahre, im zweiten Quadranten verrät B seinen Gegenspieler. Er selbst geht straffrei aus, A erhält 5 Jahre. Umgekehrt gilt diese Situation für den dritten Quadranten. Im vierten Quadranten werden schließlich beide bestraft, weil sie den anderen verraten haben.
} 
Arnold, S. - Zur Beziehung zwischen Literatur und Recht

\begin{tabular}{|c|l|l|}
\hline & \multicolumn{1}{|c|}{$\begin{array}{c}\text { Kooperation (B } \\
\text { schweigt) }\end{array}$} & \multicolumn{1}{c|}{$\begin{array}{c}\text { Defektion (B gesteht, } \\
\text { verrät A) }\end{array}$} \\
\hline Kooperation (A schweigt) & $\mathrm{A}=-2, \mathrm{~B}=-2$ & $\mathrm{~A}=-5, \mathrm{~B}=0$ \\
$\mathrm{R} / \mathrm{R}$ & $\mathrm{S} / \mathrm{T}$ \\
\hline Defektion (A gesteht, & $\mathrm{A}=0, \mathrm{~B}=-5$ & $\mathrm{~A}=-4, \mathrm{~B}=-4$ \\
verrät B) & $\mathrm{T} / \mathrm{S}$ & $\mathrm{P} / \mathrm{P}$ \\
\hline
\end{tabular}

$$
\begin{aligned}
& \mathrm{R}=\text { Reward } \\
& \mathrm{T}=\text { Temptation } \\
& \mathrm{S}=\text { Sucker's payoff } \\
& \mathrm{P}=\text { Punishment }
\end{aligned}
$$

Das Schema folgt den verschiedenen Szenarien, die Alev proleptisch beschreibt, bevor das Spiel im Roman überhaupt begonnen hat. Zunächst erwähnt er die scheinbar verführerischste Variante: den anderen $\mathrm{zu}$ verraten in der Hoffnung, straffrei auszugehen (rechts oben bzw. links unten). Dies kann aber nur gelingen, wenn der andere gleichzeitig gesteht. Üben beide Verrat, werden sie mit jeweils vier Jahren bestraft (rechts unten). Schließlich bliebe noch die Möglichkeit einer Kooperation durch wechselseitiges Schweigen (links oben).

Das Interessante an dieser Konstellation ist nun, dass die aus subjektiver Sicht scheinbar beste Strategie, nämlich den anderen zu verraten, bei einer kollektiven Betrachtungsweise nicht die optimale ist. Straffrei auszugehen kann in dieser Situation nur geschehen, wenn der andere schweigt. Wenn aber beide diese Strategie verfolgen, so trifft beide eine Haftstrafe von vier Jahren. Kollektiv betrachtet wäre das Schweigen beider die beste Alternative, da sie die geringste Gesamthaftstrafe, nämlich nur insgesamt 4 Jahre (2 Jahre für jeden) mit sich bringt. Allerdings ist in dieser Versuchsanordnung keine Kommunikation möglich.

Im vorliegenden Roman wird Smutek, Lehrer für Deutsch und Sport am ErnstBloch-Gymnasium, dazu genötigt, regelmäßigen, von einer Kamera aufgezeichneten Geschlechtsverkehr mit seiner Schülerin Ada zu vollziehen. Dies bildet den Hintergrund, vor dem sich die beiden zur Kooperation oder zur Defektion (Verrat) 
entscheiden können. Kooperieren die beiden, so besteht ihre Belohnung darin, dass beide auf dem Gymnasium bleiben dürfen. Verrät Ada Smutek und stellt sich als Opfer dar, so müsste dieser die Schule verlassen. Sie könnte damit rechnen, das Spiel beenden zu können (links unten). Gleichzeitig muss sie aber auch annehmen, dass auch sie von Smutek verraten wird und somit ihr explizit geäußerter Wunsch, Ernst-Bloch nicht verlassen zu müssen, zunichte gemacht werden könnte (rechts unten).

Die Implementierung dieses spieltheoretischen Szenarios in den Roman erlaubt nun ein Durchspielen verschiedener Möglichkeiten. Geht man davon aus, dass in literarischen Texten mögliche Welten (vgl. GOODMAN 1984: 130) geschaffen werden, so bietet die literarische Verarbeitung solcher theoretischer Überlegungen, die in der Frage nach den Handlungsmotivationen der Akteure auch dem juristischen Diskurs zu Grunde liegt, ein Spielfeld für die Ausarbeitung möglicher Handlungsketten. Die Problematik in der im Roman entworfenen Welt besteht indes darin, dass die Protagonisten frei von jeglicher Form moralischer Regelwerke sind (sie bezeichnen sich selbst als ,die Urenkel der Nihilisten“, ZEH 2004: 7). Am Ende brechen die Spieler mit den Regeln des Spiels und kommunizieren miteinander. Der abrupten Verkündung des Spielleiters Alev: "Das Spiel ist aus“ (ZEH 2004: 512) folgt, dass Smutek Alev brutal zusammenschlägt, was schließlich zur Gerichtverhandlung führt. Ein Gedankenexperiment ${ }^{20}$ an der Schnittstelle von Jura und Wirtschaftswissenschaft bildet den strukturellen Hintergrund für die im Roman entworfene Welt, ihre Figuren und deren Spielzüge. Im Roman wird ein Szenarium durchgespielt, womit im Sinne des Spielens als Probehandeln der Roman mögliche Verhaltensweisen, Kooperationen und Verkettungen zu durchdenken vermag. Das Verhalten der Spieler entspricht am Ende indes nicht den in Axelrods Schema vorgegebenen Möglichkeiten, wenn die Spieler das Spiel plötzlich unterbrechen und sich gegen den Spielleiter verbünden - die Realität der im Roman entworfenen möglichen Welt zeigt sich in ihrer Ausgestaltung damit als reicher als die zuvor in der Theorie für sie entworfene Vorhersage. Literatur fungiert hierbei als Experimentierfeld für juristische Szenarien, was in der Inszenierung einer abschließenden Gerichtsverhandlung gipfelt.

\footnotetext{
${ }^{20}$ Der Begriff des Gedankenexperiments ist in der wissenschaftlichen Diskussion umstritten. So postuliert Tobias KLAUK (2011: 32-36) einen Dreischritt für jedes Gedankenexperiment, den er insbesondere in literarischen Realisierungen nicht verwirklicht sieht. Danach folgt (a) der Vorstellung eines Szenarios (b) die spezifische Beurteilung des Szenarios mit seinen Konsequenzen und (c) eine allgemeine Beurteilung. In Spieltrieb ist es jedoch durch die explizite Einführung des Szenarios, das Durchspielen verschiedener Realisierungsmöglichkeiten sowie die Beurteilung durch die Richterin möglich, all diese Schritte zu identifizieren.
} 
1.3 Zum Verhältnis von Wort und Tatsache: ratlose Richter und Erzählrahmungen

Die Inszenierung von Gerichtsszenen gehört zu den ureigenen Ausarbeitungen juristischer Problemfelder in der Literatur. Während solche Fictions of Law (vgl. DuONG 2005: 33) in Filmen oftmals dem Hollywood-Muster einer Konfrontation von Gut und Böse folgen (vgl. MACHURA/Ulbrich 2001: 125), bei der der aufopfernde Anwalt den Sieg für das Gute davonträgt, stellen literarische Schilderungen häufig eher die Kontingenz von juristischen Entscheidungen in den Mittelpunkt. Dies geschieht im Roman Spieltrieb mithilfe einer am Ende des Romans plötzlich auftretenden IchErzählerin, „der kalten Sophie“, die dem bisher Erzählten eine Rahmung gibt und Raum lässt für die Reflexionen einer erfahrenen Richterin, die indes zunehmend an der Unentscheidbarkeit des Falles verzweifelt. Reflexionen über das Verhältnis von Recht und Gerechtigkeit stellen in der Literatur nichts Neues dar. Schon Kleists Michael Kohlhaas verzweifelt an der mangelnden Deckungsgleichheit, die auch in Ferdinand von Schirachs jüngstem Roman Tabu zentral ist. In den ausführlichen Reflexionen der in der ersten Person erzählenden Richterin heißt es im Roman Spieltrieb dazu: „Das Recht ist kein Kreißsaal für die Gerechtigkeit“ (ZEH 2004: 518). Darüber hinaus reflektiert der Roman in der Rahmung durch die Ich-Erzählerin über seine eigene Form sowie über eine mögliche Verbindungslinie zwischen Literatur und Recht, wenn es heißt:

Das Recht besteht aus Gesetzen, Gesetze bestehen aus Wörtern, und Wörter können manches sein, aber sicher nicht gerecht. Wie soll eine geschriebene Regel für unendlich viele Fallkonstellationen gedacht, angesichts der Einmaligkeit eines Geschehens eine gerechte Aussage treffen? (ZEH 2004: 518).

Die Ich-Erzählerin rekurriert hierbei auf eine zentrale Aufgabe des Juristen, die Juli Zeh so fasst, „daß sozusagen die Urtätigkeit des Juristen letztlich darin besteht, den Sinngehalt eines Wortes zu ermitteln und ihn in Beziehung zur Realität zu setzen“ (WEBER 2007: 200). Die Fallkonstellationen, für die dieses zu Grunde liegende Wort gedacht ist, bleiben stets im Konjunktiv und jeder neue in der Realität eintretende Fall bedarf eines Vermittlungsakts mit dem zu Grunde liegenden Wort bzw. Regelwerk. Der titelgebende Spieltrieb vermag genau dies in der literarischen Dimension zu leisten: ein 
Durchspielen einiger, nicht unendlich vieler, möglicher Fälle. Mithilfe der Rahmung durch eine Ich-Erzählerin kann die Vermittlung von konkretem Fall und Regel erfolgen, was indes in diesem Fall nur zur Verzweiflung der Richterin führt: so heißt es am Ende über die reflektierende Richterin, die ,,in sich hineinhorcht, um ein Urteil zu finden, und es antwortet ihm - nichts!“ (ZEH 2004: 519). Damit gleicht die Verzweiflung der Richterin dem Ausgangspunkt einer Erzählung Ingeborg Bachmanns mit dem Titel Ein Wildermuth, in der der gleichnamige Protagonist und Richter an der Unentscheidbarkeit des Falles verzweifelt und schreiend das Gericht verlässt. Auch hier wird, nachdem die Geschichte in Grundzügen von einem heterodiegetischen Erzähler wiedergegeben wird, eine Ich-Erzählung des Richters eigeschoben, der über das Verhältnis von Wahrheit und Tatsachen in der Welt reflektiert: „Wahrheit ist nur zur Hälfte Menschenwerk, denn es muß ihr auf der anderen Seite etwas entsprechen, dort, wo die Tatsachen sind“" (BACHMANN 1975: 171). Die Vermittlung zwischen Wort und Tat ist eine der zentralen Aufgaben der Justiz, die in der Literatur thematisiert werden und in ihrer Fähigkeit als System der „Beobachtung zweiter Ordnung“ (LuHMANN 1995: 339) reflektiert werden kann. Durch ihre Systemeigenschaft der Möglichkeit zur Autoreflexivität vermag sie die Problematik einer auf sich selbst rekurrierenden Sprache darzustellen. Der Literatur bleibt hierbei auch die Darstellung des Scheiterns vorbehalten, wie WEITIN (2010: 36) für Kleists Der Zerbrochene Krug nachweist, das sich im Fall von Ingeborg Bachmann und Juli Zeh in der mangelnden Deckungsgleichheit von Wort und Tat und im Misslingen einer sprachlich vermittelten Gerechtigkeitsvorstellung zeigt. Der Roman vermag es, die Kontingenz bestimmter Gerechtigkeitsvorstellungen sowie die grundsätzliche Anlage des Rechts als sprachliche Struktur, die nichtsprachlicher Konstellationen Herr $\mathrm{zu}$ werden versucht, darzustellen. Die herausgearbeiteten Funktionen spielen für die Berührungspunkte zwischen Literatur und Recht insbesondere auch eine große Rolle, wenn die Wechselwirkungen zwischen den beiden Diskursen im Mittelpunkt stehen, und hängen wesentlich mit den jeweiligen Rezeptionshaltungen ab. Diese beiden Aspekte werden im Folgenden für zwei Texte Ferdinand von Schirachs in den Blick genommen. 
Arnold, S. - Zur Beziehung zwischen Literatur und Recht

\section{Ferdinand von Schirach: Verbrechen (2009), Tabu (2013)}

\subsection{Die Rolle von Gattung und Erzählinstanz: Moderne Pitavalgeschichten}

Mit seinem literarischen Erstlingswerk, der Kurzgeschichtensammlung Verbrechen, gelang dem Autor und Strafverteidiger Ferdinand von Schirach 2009 ein internationaler Erfolg. Die Geschichten, die im Paratext unter der englischsprachigen Gattungsbezeichnung Stories geführt werden, wurden in mehr als dreißig Sprachen übersetzt und für Bühne und Fernsehen adaptiert. ${ }^{21}$ Den Hintergrund für diesen Erfolg bilden elf Geschichten über Verbrechen und Gerichtsverfahren, die in der Tradition der Pitavalgeschichte die Lebensgeschichte des Täters, seine Psyche und sein soziales Umfeld mit Bezug zur Tat einschließen. Insbesondere im 19. Jahrhundert galt diese Form der Geschichte als ein wichtiges Instrument der Rechtswissenschaft (vgl. LACHENMAIER 2008: 18 f.). Erzählt werden auch bei Schirach neben dem eigentlichen Verbrechen, das meist schnell rekapituliert ist, Motive, Hintergründe und der Weg des Täters zu seinem Verbrechen. ${ }^{22}$ Inwiefern es sich dabei um eine faktuale Textsorte handelt, lässt sich nicht eindeutig bestimmen; vielmehr macht Schirach selbst darüber immer wieder widersprüchliche Aussagen. ${ }^{23}$ Wenngleich aufgrund der rechtlichen Lage zum Schutz der Privatsphäre der Betroffenen deutlich sein müsste, dass es sich bestenfalls um eine faktuale Basis handeln kann (wie Schirach selbst auch immer wieder betont), ${ }^{24}$ lässt sich doch in der Rezeption der Texte die Tendenz beobachten, dass diese als faktual gelesen werden, so etwa in der Kritik Denis Schecks, der

\footnotetext{
${ }^{21}$ Hierunter fallen die gleichnamige TV-Serie Verbrechen für das ZDF (2013), produziert von Oliver Berben, die bereits nach Taiwan exportiert wurde, die Verfilmung der Kurzgeschichte Glück durch Doris Dörrie (2012) sowie eine dramaturgische Fassung in Japan am Tokyo Metropolitan Theater 2013.

${ }^{22}$ Vgl. den Kommentar des Spiegel-Rezensenten Arno Frank zum Erscheinen der TV-Serie Verbrechen: „Es geht nicht darum, einen Täter zu überführen - es geht darum, ihn zu verstehen.“ http://www.spiegel.de/kultur/tv/edelkrimi-serie-verbrechen-im-zdf-nach-ferdinand-von-schirach-a892075.html (27/01/2014).

${ }^{23} \mathrm{Vgl}$. Schirachs Interview mit Alexander Kluge: http://www.dctp.tv/\#/filme/ferdinand-von-schirachverbrechen-aus-dem-alltag-eines-strafverteidigers/ (27/01/2014) und seine Rede zur Verleihung des Kleist-Preises: ,ja, die Geschichten sind ganz und gar wahr. Aber sie sind nicht wahr, weil sie der Realität entsprechen, sie sind wahr, weil sie Literatur sind.“ www.tagesspiegel.de/kultur/rede-zum-kleistpreisjeder-kann-zum-moerder-werden/3088620.html (27/01/2014).

${ }_{24}$ „Also, die Fälle sind ja nicht $1: 1$, das darf ich ja nicht schreiben. Aber in der Geschichte war es so (...).“ http://www.ndr.de/fernsehen/sendungen/das/videos/dasx4213.html [27.01.2014].
} 
Schirachs Geschichten als „geborgtes Leben“ aburteilte. ${ }^{25}$ Motto und Klappentext tun ein Übriges, um diese Rezeptionshaltung zu fördern. Während im Klappentext die Geschichten als „unglaubliche Geschichten, doch sie sind wahr“ bezeichnet werden, referiert das Motto auf die Heisenbergsche Unschärferelation, ${ }^{26}$ einer von Schirachs zentralen Einflüssen, ${ }^{27}$ der sich auch im Roman Tabu fortsetzt, ${ }^{28}$ und in Schirachs Interpretation die Grenze zwischen Wahrheit und Fiktion durchlässig macht. Wenn auch der faktuale Gehalt der Geschichten zweifelhaft ist und bislang von Presse und Kritik noch nicht nachgewiesen werden konnte - ein 72-jähriger Mediziner aus Rottweil, der seine Frau im Keller mit der Axt erschlagen hat, müsste durch entsprechende Presseberichte leicht ausfindig zu machen sein - so spielt Schirach doch zunächst durch die Einpassung seiner Geschichten in die Tradition des faktualen Genres der Pitavalgeschichten, dann aber auch durch widersprüchliche para- und außertextuelle Angaben mit der Rezeptionshaltung des Lesers. Wichtig ist diese in Bezug auf die Funktionen transjuristischer Darstellungen, insofern die Tradition der Pitavalgeschichte als faktuale Textsorte, einen Lernprozess über die Realität impliziert. ${ }^{29}$ Schirach, so die These dieses Beitrags, nutzt diese Tradition, um Aussagen über die Realität der Rechtsprechung zu treffen oder zumindest nahezulegen. Hierfür muss der Text nicht notwendig als faktualer identifiziert werden, er kann trotz seines faktualen Status durch die genannte Strategien Hypothesen über die reale Welt enthalten oder den Leser zur Formung solcher Hypothesen anregen (vgl. GITTEL 2014). ${ }^{30}$

Erzählt werden die Geschichten von einem zunächst heterodiegetisch erscheinenden Erzähler, der jedoch jeweils am Ende der Geschichte als homodiegetischer Ich-Erzähler ${ }^{31}$ und mit dem Fall betrauter Rechtsanwalt erkennbar wird. Dabei ist nicht zu entscheiden, inwiefern das vorher Erzählte, das die Ereignisse

\footnotetext{
${ }^{25}$ http://www.tagesspiegel.de/kultur/aufgeschlagen-zugeschlagen-unsichtbarefolterknechte/1913428.html (27/01/2014).

26 „Die Wirklichkeit, von der wir sprechen können, ist nie die Wirklichkeit an sich.“

$27 \mathrm{http} / / / \mathrm{www}$.tagesspiegel.de/kultur/rede-zum-kleistpreis-jeder-kann-zum-moerderwerden/3088620.html (27/01/2014).

${ }^{28}$ So beispielsweise im als Hinweis gekennzeichneten Zusatz am Ende des Romans: „Die Ereignisse in diesem Buch beruhen auf wahren Begebenheiten. >>Wirklich?<<, fragte Biegler." (SCHIRACH 2013: 256).

${ }^{29}$ Im 19. Jahrhundert wurden die Pitavalgeschichten zum einen als Fallbeispiele in der juristischen Ausbildung verwendet, zum anderen als Aufklärung eines Laienpublikums (vgl. LACHENMAIER 2008: 19).

${ }^{30}$ Auch wenn es sich um fiktionale Sätze handelt, die streng genommen nicht auf die Realität bezogen werden können, können vom fiktionalen Werk ausgehend Hypothesen über die reale Welt gebildet werden. Man spricht dann von Bedeutungsrelationen oder Kausalrelationen (vgl. HosPERS 1960: 43 f.).

${ }^{31}$ Dies ist jedoch für fiktionale Texte nicht ungewöhnlich, wird der Übergang zwischen Homodiegese und Heterodiegese von Erzähltheoretikern gemeinhin als graduell begriffen (vgl. ZIPFEL 2001: 132).
} 
Arnold, S. - Zur Beziehung zwischen Literatur und Recht

vor der Gerichtsverhandlung umfasst und formal meist durch einen Geviertstrich abgetrennt ist, als in den Rahmen der Ich-Erzählung eingebettet zu betrachten ist, ${ }^{32}$ wenngleich bestimmte Formulierungen wie „Die drei jungen Männer erzählten mir die Geschichte“ (SCHIRACH 2009: 36), darauf hindeuten, dass sich die Erzählinstanz nicht ändert und hier lediglich erstmalig, angezeigt durch ein Pronomen, explizit in Erscheinung tritt. Markiert wird die Beurteilung und Informationsvergabe des Geschehenen durch die Augen des Anwalts also erst am Ende der Erzählung, jedoch lassen sich bereits vorher deutlich wertende Urteile des Erzählers im Text identifizieren. Der Vorwurf der Kritik an Schirachs Roman Tabu, der Erzähler mische sich belehrend ein, ${ }^{33}$ gilt - wenn auch weniger explizit - bereits für den Erzählband Verbrechen. Einerseits gelingt dies durch den, durchaus dem Genre der Pitavalgeschichte entsprechenden, einseitigen Einblick in die Gedankenwelt einer Figur durch indirekte Gedankenwiedergabe oder erlebte Rede (,Er erschrak und nahm sie in die Arme. Auf seiner Brust spürte er ihren Herzschlag, er war hilflos. Sie ist mir anvertraut, dachte er", SCHIRACH 2009: 10, „ein Versprechen nur für gute Tage gab es nicht“, ebd.: 13 f.), andererseits durch explizite Meinungsäußerungen des erzählenden Rechtsanwalts. ${ }^{34}$ Dieser spielt sowohl in der literarischen Vorlage als auch in der ZDF-Serie eine entscheidende Rolle und ist auf dem Buchcover und im Vorspann der ZDF-Serie sowie jeweils im Abspann der einzelnen 45-minütigen Episoden zu sehen. Dort schreitet er nach einem schnellen Zusammenschnitt verschiedener Bilder von Leichen, Überfällen, Berlinversatzstücken, Polizeieinsätzen und Gerichtsverfahren souverän auf die Kamera $\mathrm{zu}$.

In der Gerichtsverhandlung der den Erzählband einleitenden Geschichte Fähner kommt es schließlich zu einer solchen expliziten Meinungsäußerung des Anwalts mit anschließenden juristischen und rechtsphilosophischen Erklärungen. Der 72-jährige Arzt Friedhelm Fähner hat nach 48 Ehejahren seine Ehefrau im Keller mit einer Axt

\footnotetext{
${ }^{32}$ Die ZDF-Serie geht jedoch hierbei vom Ende aus. Beginnend mit dem Mord Fähners an seiner Frau wird im Verhörzimmer, wo sich Fähner und sein Anwalt zum ersten Mal treffen, in der Retrospektive erzählt.

33 „Aber während Leonardt die literarische Verkörperung juristischer Neutralität darstellte, darf Biegler durch den Fall poltern, um noch dem letzten Stümper vor und hinter dem Gericht klar zu machen, welche Spiel-Räume das Rechtssystem bietet, ohne dass dafür philosophische Pirouetten gedreht werden müssen.“ http://www.welt.de/kultur/literarischewelt/article120871701/Stilvoller-Weltschmerz-mitFerdinand-von-Schirach.html [27.01.2014].

${ }^{34}$ Diese Einflussnahme wird von Schirach jedoch bestritten, beispielsweise wenn er im Interview des Zusatzmaterials der ZDF-Serie Verbrechen konstatiert, der Zuschauer befinde sich „auf Augenhöhe“ und „er wählt keine Seite, sondern ist ein Beobachter“.
} 
Arnold, S. - Zur Beziehung zwischen Literatur und Recht

erschlagen. Dem voraus ging ein langjähriges Martyrium des von seiner Ehefrau zunehmend unterdrückten und in seiner Freiheit eingeschränkten Fähners. Gerade dieser Situation konnte sich Fähner, so die Argumentation des Rechtsanwalts, nicht entziehen, weil er als vormoderner Mensch an ein Versprechen aus den Flitterwochen sowie das Ehegelübde gebunden war - so kann der Ich-Erzähler und Rechtsanwalt die Position des Staatsanwalts deutlich aburteilen: „Der Staatsanwalt irrte, genau das hätte Fähner nicht gekonnt" (SCHIRACH 2009: 17). Diese Stellen werden darüber hinaus für juristische Belehrungen genutzt, die der Leser, unabhängig davon, ob er die Textsorte als faktual klassifiziert, auf die Realität beziehen kann. An dieser Stelle geht es um die urteilsentscheidende Frage, ob Fähner eine Alternative gehabt hätte. Diese hätte laut Staatsanwalt in einer Scheidung von seiner Ehefrau bestanden. Der Rechtsanwalt und Ich-Erzähler bedient sich einer zweigleisigen Verteidigungsstrategie. Zunächst nimmt er immer wieder auf die Kraft des Versprechens und seine Bedeutung für Fähner Bezug, um so zu zeigen, dass die Alternative einer Scheidung nicht bestanden hätte: „Fähner konnte sich nicht befreien, das wäre Verrat gewesen“" (SCHIRACH 2009: 17). Anschließend folgen rechtsphilosophische Überlegungen über den Sinn des Strafens und die Anwendung auf den vorliegenden Fall, die Grundlagen des Rechtssystems für den Laien aufbereiten und das anschließende Urteil (Fähner kann seine Strafe im offenen Vollzug verbüßen) erklären:

Es gibt eine Fülle von Theorien. Strafe soll uns abschrecken, Strafe soll uns schützen, Strafe soll den Täter davon abhalten, nochmals eine Tat zu begehen, Strafe soll Unrecht aufwiegen. Unser Gesetz vereinigt diese Theorien, aber keine passte hier richtig. Fähner würde nicht erneut töten. Das Unrecht der Tat war offensichtlich, aber es war schwer zu wiegen. Und wer wollte Vergeltung üben? (SCHIRACH 2009: 17).

Neben dem belehrenden Charakter, den diese Passage enthält, weist sie deutlich auf eine Lücke im Rechtssystem bzw. auf einen in der Realität (der fiktionalen Welt) eintretenden Fall hin, der von den Gesetzgebern nicht vorgesehen wurde. Sowohl Schirachs widersprüchliche Aussagen über den faktualen Charakter der Texte als auch die Einschreibung in die Tradition der Pitavalgeschichte fördern eine Rezeptionshaltung, die die ursprünglichen Funktionen der Pitavalgeschichte beibehält. Zum einen unterhalten sie mit Fällen, die real oder auf die Realität bezogen sind, zum anderen haben sie einen didaktischen Charakter und belehren den Leser über die Funktionsweise des Rechtssystems. Literatur kann dabei aktiv an nichtliterarischen 
Arnold, S. - Zur Beziehung zwischen Literatur und Recht

Diskursen teilnehmen, indem sie bestimmte Vorstellungen von Recht und Gerechtigkeit transportiert (vgl. FLUDERNIK/OLSON 2004: xviii) oder aktuelle Diskurse aufnimmt und sie ihren systeminternen Eigenschaften entsprechend verarbeitet.

\subsection{Die Würde ist antastbar - Fiktionalisierung eines politischen Manifests}

Im September 2013 veröffentlichte Ferdinand von Schirach einen Aufsatz im Spiegel mit dem Titel Die Würde ist antastbar, der später in einer Übersetzung auch in der spanischen Zeitung El País erschien. ${ }^{35}$ Im gleichen Jahr erschien sein Roman Tabu, der obgleich er an einigen Stellen einem Künstler- oder Entwicklungsroman ähnelt, sich mit Schirachs Grundfragen nach Schuld und Bestrafung befasst und zahlreiche Referenzen zu Schirachs nichtliterarischem Manifest im Spiegel aufweist. Sebastian von Eschburg, der aufgrund der mangelnden Liebe seiner Eltern, deren späterer Scheidung und dem Selbstmord des Vaters eine schwere Kindheit verbringt und schon früh zu Schwermut, gleichzeitig aber auch künstlerischer Sensibilität neigt, wird Fotograf, wobei ihn primär der Unterschied zwischen Wahrheit und Wirklichkeit beschäftigt. In einem einmaligen Performance-Projekt lässt er eine vermeintlich von ihm entführte Frau bei der Polizei anrufen. Sie befinde sich in Eschbachs Gewalt und sei im Kofferraum eines Autos mit unbekanntem Aufenthaltsort eingeschlossen. Sofort beginnen die polizeilichen Ermittlungen und Eschbach kommt in Untersuchungshaft. Bei der Untersuchung entsteht ein Phantombild, das, wie sich später herausstellt, jedoch nur eine künstlerische Collage aus drei verschiedenen Gesichtern ist; die eigentliche Entführung wurde hingegen lediglich inszeniert und die zugehörige Entführte existiert nicht. Brisant wird der Fall dadurch, dass ein Polizist Eschburg Folter androht, um den Aufenthaltsort der vermeintlich Entführten zu erfahren und damit das titelgebende Tabu bricht. Dieser Fall erinnert an reale Fälle, der bekannteste unter ihnen der Magnus Gäfgen-Fall, ${ }^{36}$ Entführer und Mörder des Bankierssohns Jakob von Metzler, der später einen Prozess wegen einer Folterdrohung in Haft führte.

Neben den Parallelen zwischen literarischem und nichtliterarischem Text werden für die Bestimmung der Relation zwischen Literatur und Recht vor allem die Unterschiede relevant. So erhält die Figur des ermittelnden Anwalts Biegler zunächst

\footnotetext{
${ }^{35}$ http://www.spiegel.de/spiegel/print/d-112638562.html (27/01/2013).

${ }^{36}$ Auf diesen referiert Schirach auch in seinem Spiegel-Aufsatz.
} 
Raum für zahlreiche Reflexionen über das Verhältnis von Folter und Menschenwürde und kann sich sodann belehrend dem betroffenen Polizisten gegenüber äußern: „Sie haben sich gegen unsere Rechtsordnung gestellt, gegen alles, woran ich glaube. Sie haben die Würde eines Menschen verletzt. Diese Würde kann ein Mensch nicht erwerben und er kann sie nicht verlieren.“ (SCHIRACH 2013: 230). Biegler äußert hier Gedanken, die auch in Schirach Aufsatz über die Folter vorkommen, in dem es ebenfalls um die Frage geht, ob die Würde eines einzelnen zur Rettung vieler unter Umständen eingeschränkt werden kann: „Der Staat kann ein Leben niemals gegen ein anderes Leben aufwiegen. Keiner kann wertvoller sein als ein anderer, eben weil Menschen keine Gegenstände sind. Und das gilt auch für große Zahlen.“37 Zunächst scheinen die Aussage Schirachs und diejenige des fiktionalen Bieglers deckungsgleich. Während sich Schirach innerhalb der Argumentation des faktualen Textes einer Vielzahl imaginativer Szenarien bzw. Gedankenexperimenten bedient, kann in der Fiktion ein solches jedoch viel detaillierter und vollständiger ausgearbeitet werden. Am Ende des Romans wird enthüllt, dass ein Unschuldiger gefoltert wurde. Der epistemisch ohnehin bereits privilegierte Biegler erhält somit ein weiteres Argument für sein Plädoyer gegen die Folter. Inwiefern dieses schlüssig ist, soll noch diskutiert werden. Zunächst lässt sich jedoch festhalten, dass sich durch die Zuordnung von Gedanken zu einer Romanfigur, die im Figurengefüge epistemisch privilegiert ist, dieser gewisse Autorität verleihen lässt. Ähnlich wie in den essayistischen Passagen bei Juli Zeh können durch die detaillierten Äußerungen des Rechtsanwalts in der Gerichtsverhandlung juristische Probleme vom Erzähler oder von einzelnen Figuren verhandelt und bewertet werden. Dies allein wäre indes noch eine zu einfache Erklärung für die Vielzahl transjuristischer Darstellungen in der Gegenwartsliteratur.

Ein weiterer Aspekt ist in der Rolle der Literatur als „Unfallschauplatz“ (WEITIN 2010: 36) zu finden. Beim „Entwurf möglicher Welten“ (LÜDERSSEN 1991: 12 f.) können theoretische Probleme durchdacht und in verschiedenen Szenarien durchgespielt werden. Für die Rechtswissenschaft spielen solche Gedankenexperimente eine grundlegende Rolle. So kennt jeder Jura-Student das Beispiel der Höhlenforscher, The Case of the Speluncean Explorers, von Lon L. Fuller, bei dem fünf Höhlenforscher in Not geraten und einen aus ihrer Mitte opfern, um ihr Überleben zu sichern. Zu den rechtlichen Lösungen des Falls und den juristischen Konsequenzen gibt es mehrere

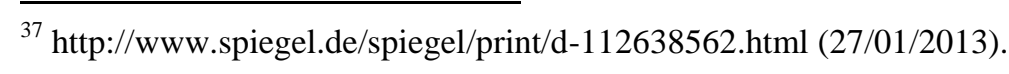

Pandaemonium, São Paulo, v. 17, n. 23, Jun./2014, p. 1-25 
Arnold, S. - Zur Beziehung zwischen Literatur und Recht

mögliche Szenarien und theoretische Überlegungen. Dieser konjunktivischen Gedankenspiele bedient sich auch Schirach in seinem Manifest, ${ }^{38}$ sie sind aber auch im Sinne einer Bezugnahme der fiktionalen Welt auf die reale Welt als Wenn $x$ in der realen Welt geschehen würde, was wären die Konsequenzen? auf die aktuellen Diskurse übertragbar. Sowohl im Gedankenexperiment in Tabu als auch in den von Schirach in seinem Manifest verwendeten Gedankenspielen (Die Königin gegen Dudley und Stephens) und dem Höhlenbeispiel geht es primär um die Frage, ob unter bestimmten Umständen die Würde des einzelnen zur Rettung vieler antastbar ist. Der Roman bietet im Sinne eines solchen Gedankenexperiments den Raum, ein komplettes hypothetisches Szenario auszuarbeiten und in der Gerichtsverhandlung verschiedene Positionen einander gegenüberzustellen. Am Ende stellt sich heraus, dass es nie ein Verbrechen gegeben hat und somit ein Unschuldiger gefoltert wurde. Es wird damit eine vergleichsweise einfache Lösung gewählt. Der Protagonist, Rechtsanwalt Biegler, spricht sich gegen Folter aus und begründet dies wahlweise damit, dass ausgeschlossen werden muss, dass Unschuldige gefoltert werden oder mit einem generellen Folterverbot. Das Szenario, in einer solchen Situation (durch Folter eines einzelnen könnte das Leben von vielen gerettet werden) auch einem Schuldigen die Würde abzuerkennen, wogegen sich Schirach zumindest in seinem nichtliterarischen Text wendet, wird im Roman nicht bedacht.

\section{Zusammenfassung}

Am Beispiel von Juli Zehs Roman Spieltrieb und Ferdinand von Schirachs Verbrechen und Tabu wurde, unter Berücksichtigung ihrer medialen Adaptionen, die Beziehung zwischen Literatur und Recht in der deutschsprachigen Gegenwartsliteratur untersucht. Dabei standen insbesondere die ästhetischen Besonderheiten, Funktionen literarischer Rechtsdarstellungen sowie die Wechselwirkungen zwischen literarischem und juristischem Diskurs im Mittelpunkt.

Die Dominanz essayistischer Passagen, die als Gedächtnis der Literatur an den essayistischen Roman erinnern, erlaubt bei Juli Zeh eine Vielzahl von Reflexionen von

\footnotetext{
${ }^{38}$ Am Beispiel des Falls Die Königin gegen Dudley und Stephens stellt Schirach die Frage: „Durften die Seeleute den Schiffsjungen töten, um ihr eigenes Leben zu retten? Drei Leben gegen eines. Das Gericht sollte darüber urteilen, ob eine solche Rechnung erlaubt ist.“ http://www.spiegel.de/spiegel/print/d112638562.html (27/01/2013)
} 
Arnold, S. - Zur Beziehung zwischen Literatur und Recht

Erzähler und Figuren zu rechtlichen Fragestellungen. Das Überwiegen von subjektiven und experimentellen Elementen im essayistischen Roman korrespondiert mit dem im Anschluss an Robert Musil ausgearbeiteten Begriffspaar Möglichkeit und Wirklichkeit und bildet die Funktionsweise des Rechts in der Tradition des code law systems ab, das in seiner Ausformulierung auf alle mögliche Szenarien anwendbar sein soll. In der Fiktion können solche Szenarien durchgespielt und weitergedacht werden. Dies geschieht in Spieltrieb am Beispiel des aus der Spieltheorie entlehnten Gefangenendilemmas, bei dem in Anlehnung an wirtschaftswissenschaftliche Theorien zunächst verschiedene mögliche Szenarien formal beschrieben werden. Im Roman werden die Figuren zu Spielern, ihre Handlungen zu Spielzügen innerhalb dieses Modells. Jedoch endet das Dilemma anders als in den theoretischen Beschreibungen vorgesehen. Im komplexeren literarischen Experimentierfeld kommen somit weitere Möglichkeiten hinzu, die im theoretischen Modell ausgeschlossen bleiben. Die Beurteilung obliegt einer Richterin, die sich als Ich-Rahmenerzählerin in den Roman einschaltet und deren Aufgabe primär in der Vermittlung zwischen geschriebenem Recht und realen Rechtsfällen besteht, die nach Juli Zeh zur genuinen Aufgabe eines Juristen gehört. In der literarischen Ausgestaltung dieser ihr zunehmend schwer fallenden Aufgabe können solche Prozesse zunächst beobachtet werden, Literatur kann als autoreflexives System die auf sich selbst bezogene Sprache darstellen, deren Vermittlung mit der Realität zunehmend schwerfällt und sie kann das Misslingen einer sprachlich vermittelten Gerechtigkeitsvorstellung sowie des Vermittelns zwischen Sprache und konkretem Fall modellieren.

Für die Wechselwirkungen zwischen den Diskursen spielen auch die jeweiligen Rezeptionshaltungen eine wesentliche Rolle. Diese sind in Schirachs Verbrechen durch die Äußerungen des Autors, paratextuelle Angaben (Klappentext, Motto) sowie durch den Bezug auf das faktuale Genre der Pitavalgeschichte als auf die Realität beziehbar angelegt. Dem Genre der Pitavalgeschichte entsprechend sollen die Geschichten einerseits unterhalten, andererseits einen Lernprozess initiieren. Dementsprechend ist auch die Erzählposition gestaltet, die in Form eines Ich-Erzählers, der gleichzeitig Rechtsanwalt ist, sich aber erst jeweils am Ende der Geschichten zu erkennen gibt, Kommentare und Bewertungen abgibt und den Leser über rechtliche Sachlagen belehrt. Auch im Fall von Schirach treten dabei in der fiktionalen Welt Fälle auf, die von der Gesetzgebung nicht vorgesehen wurden und die als Spezifikum einer 
Arnold, S. - Zur Beziehung zwischen Literatur und Recht

bestimmten fiktionalen Verarbeitung rechtlicher Fragestellungen bestimmt werden können. Die Parallelen zu aktuellen Diskursen wurden anhand der Frage nach der absoluten Gültigkeit der Menschenwürde erörtert, die Schirach sowohl theoretisch als auch in seinem jüngsten Roman Tabu in den Mittelpunkt stellt. Die zentrale Rolle von Gedankenexperimenten für die Rechtswissenschaft kann dabei zum Ansatz genommen werden, um in der Literatur weitere Ausarbeitungen entsprechender Szenarien zu bestimmen. Es werden in einer literarisch inszenierten Gerichtsverhandlung verschiedene Positionen einander gegenübergestellt, wobei durch die Vergabe einer bestimmten Position an eine im Figurengefüge epistemisch privilegierte Figur sowie durch Lenkung des Szenarios in eine bestimmte Richtung (ein Unschuldiger wird geopfert) auch Rezeptionshaltungen beeinflusst werden können.

Die herausgearbeiteten Punkte machen deutlich, dass literarische Rechtsschilderungen eine wichtige Rolle in der Gegenwartsliteratur spielen, die vergleichsweise spezifische Funktionen erfüllen, die wiederum mit bestimmten ästhetischen Verfahren verbunden sind. Literatur bildet den rechtlichen Diskurs dabei nicht lediglich ab, sondern partizipiert, ihren systemeigenen Funktionen entsprechend, an der gegenwärtigen Verhandlung rechtlicher Fragestellungen.

\section{Literaturverzeichnis}

ARNOLD, Sonja. Zwischen Nihilismus und Spieltheorie. Juli Zehs Roman Spieltrieb. In: Revista de Filología Alemana 19, 2011, 207-222.

AXELROD, Robert. Die Evolution der Kooperation, 6. Aufl. München, Oldenbourg, 2005.

BALKIN, Jack M. / LEVINSON, Sanford. Law and the Humanities: an Uneasy Relationship. In: 18 Yale Journal of Law and the Humanities 2, 2006, 155-186.

BACHMANN, Ingeborg. Ein Wildermuth. In: Dies. Das dreißigste Jahr. Erzählungen. München: Piper, 1975, 157-175.

BARON, Jane B. Baron / EPSTEIN, Julia. Is law narrative? In: 45 Buffalo Law Review, 1997, 141187.

BRUGGER, Winfried / SARLET, Ingo Wolfgang. Moderner Konstitutionalismus am Beispiel der US-Verfassung, des Grundgesetzes und der brasilianischen Verfassung: eine rechtsvergleichende Perspektive. In: Jahrbuch des Öffentlichen Rechts der Gegenwart 56, 2008, 613-638.

CARDOZO Benjamin Nathan. Law and Literature. In: Ders. Selected Writings of Benjamin Nathan Cardozo. New York: Fallon Law Book Company, 1947. 
Arnold, S. - Zur Beziehung zwischen Literatur und Recht

Duong, Wendy Nicole. Law is Law and Art is Art and Shall the Two Ever Meet? Law and Literature: the Comparative Creative Processes. In: Southern California Interdisciplinary Law Journal 15, 2005, 1-43.

ERLL, Astrid / NÜNNING, Ansgar. Literaturwissenschaftliche Konzepte von Gedächtnis: Ein einführender Überblick. In: Dies. (Hg.). Gedächtniskonzepte der Literaturwissenschaft. Theoretische Grundlagen und Anwendungsperspektiven. Berlin: de Gruyter, 2005, 1-9.

GITTEL, Benjamin. Understanding the Evil with Truman Capote, Alfred Döblin and Ferdinand von Schirach. Claim and Reality of the 'Nonfiction Novel' and Related Text Types. In: ARNOLD, Sonja / KorfMANN, Michael (Hg.): Law and Literature at the Turn of the Millennium. Porto Alegre: Dublinense [erscheint 2014].

Fludernik, Monika / Olson, Greta (Hg.). In the Grip of the Law. Trials, Prisons and the Space Between. Frankfurt/Main et al.: Lang, 2004.

Goodman, Nelson. Weisen der Welterzeugung. Frankfurt/Main: Suhrkamp, 1984.

HOSPERS, John. Implied Truths in Literature. In: The Journal of Aesthetics and Art Criticism 29 (1), 1960, 37-46.

KLAUK, Tobias. Thought Experiments and Literature. In: BIRKE, Dorothee / BUTTER, Michael / KÖPPE, Tilmann (Hg.). Counterfactual Thinking / Counterfactual Writing. Berlin; Boston: de Gruyter, 2011, 30-44.

LACHENMAIER, Birgit Maria. Die Law as Literature-Bewegung - Entstehung, Entwicklung und Nutzen. Berlin: wvb, 2008.

LÜDERSSEN, Klaus. Produktive Spiegelungen. Recht und Kriminalität in der Literatur. Frankfurt/Main: Suhrkamp, 1991.

LuHMann, Niklas. Das Recht der Gesellschaft. Frankfurt/Main: Suhrkamp, 1995.

MACHuRA, Stefan / Ulbrich, Stefan. Law in Film: Globalizing the Hollywood Courtroom Drama. In: Journal of Law and Society 28 (1), 2001, 117-132.

MÖLK, Ulrich (Hg.). Literatur und Recht. Literarische Rechtsfälle von der Antike bis in die Gegenwart. Göttingen: Wallstein, 1996.

MÜLLER-DIETZ, Heinz. Grenzüberschreitungen. Beiträge zur Beziehung zwischen Literatur und Recht. Baden-Baden: Nomos, 1990.

MusIL, Robert. Der Mann ohne Eigenschaften. Erstes und zweites Buch, hg. von Adolf Frisé, 20. Aufl. Reinbek bei Hamburg: Rowohlt, 2005 [1930].

NeuHaus, Stefan. Das Subversive des Spiels: Überlegungen zur Literatur der Postmoderne. In: ANZ, Thomas / KAULEN, Heinrich (Hg.). Literatur als Spiel: Evolutionsbiologische, ästhetische und pädagogische Konzepte. Berlin: de Gruyter, 2009, 371-390.

OLSON, Greta. De-Americanizing Law and Literature Narratives: Opening Up the Story. In: Law \& Literature 22 (2), 2010, 338-364.

OLSON, Greta. Intersections of Gender and Legal Culture in Two Women Judge Shows: Judge Judy and Richterin Barbara Salesch. In: PETERSEN, Hanne / LORENZO Villaverde / LUND-ANDERSEN, Ingrid (Hg.). Contemporary Gender Relations and Changes in Legal Cultures. Kopenhagen: DJØF Publishing, 2013, 29-58.

SAMPAIO DE MoRAES Godoy, Arnaldo. Direito e literatura: Os pais fundadores John Henry Wigmore, Benjamin Nathan Cardozo e Lon Fuller. In: TRINDADE, André Karan / SCHWARTZ, Germano (Hg.). Direito e Literatura: O encontro entre Themis e Apolo. Curitiba: Juruá, 2008, 23-29.

SCHÄRF, Christian. Geschichte des Essays. Von Montaigne bis Adorno. Göttingen: Vandenhoeck \& Ruprecht, 1999.

SCHLAFFER, Heinz. Essay. In: WeIMAR, Klaus u.a. (Hg.). Reallexikon der deutschen Literaturwissenschaft, Band I. Berlin; New York: de Gruyter, 1997, 522-525.

SCHIRACH, Ferdinand von. Verbrechen. München: Piper, 2009.

Pandaemonium, São Paulo, v. 17, n. 23, Jun./2014, p. 1-25 
Arnold, S. - Zur Beziehung zwischen Literatur und Recht

SCHIRACH, Ferdinand von. Tabu. München: Piper, 2013.

SCHÖNERT, Jörg. Erzählte Kriminalität. Zur Typologie und Funktion von narrativen Darstellungen in Strafrechtspflege, Publizistik und Literatur zwischen 1770 und 1920. Vorträge zu einem interdisziplinären Kolloquium, Hamburg, 10.-12. April 1985. Tübingen: Niemeyer, 1991.

WeBER, Hermann (Hg.). Literatur, Recht und Musik. Tagung im Nordkolleg Rendsburg vom 16. bis 18. September 2005. Berlin: BWV, 2007.

WeITIN, Thomas. Recht und Literatur. Münster: Aschendorff, 2010.

ZEH, Juli. Spieltrieb. 2. Aufl. Frankfurt/Main: Schöffling \& Co, 2004.

ZEH, Juli: A Menina sem Qualidades. Trad. Marcelo Backes, Rio de Janeiro: Record, 2009.

ZEH, Juli: Schilf. 5. Aufl. München: btb, 2009.

ZEH, Juli / OsWALD, Georg M. Aufgedrängte Bereicherung. Tübinger Poetik-Dozentur 2010, hg. von Dorothee Kimmich und Philipp Ostrowicz. Künzelsau: Swiridoff, 2011.

ZIPFEL, Frank. Fiktion, Fiktivität, Fiktionalität. Analysen zur Fiktion in der Literatur und zum Fiktionsbegriff in der Literaturwissenschaft. Berlin: Erich Schmidt, 2001.

Weblinks:

ALANYALI, Iris. Stilvoller Weltschmerz mit Ferdinand von Schirach. In: Die Welt, 14.10.2013: http://www.welt.de/kultur/literarischewelt/article120871701/Stilvoller-Weltschmerzmit-Ferdinand-von-Schirach.html (27/01/2014).

Bibliographie des European Network for Law and Literature: http://www.esl.eur.nl/fileadmin/ASSETS/frg/arw/rechtstheorie/Law_Literature_Bibli ography_Greta_Olson_Jeanne_Gaakeer.pdf (27/01/2014).

Demokratie im digitalen Zeitalter. In: $\quad$ FAZ, 10.12 .2013 : http://www.faz.net/aktuell/feuilleton/buecher/themen/autoren-gegenueberwachung/demokratie-im-digitalen-zeitalter-der-aufruf-der-schriftsteller12702040.html (27/01/2014).

FRANK, Arno. Edel-Krimiserie „Verbrechen“ im ZDF: Das ist aber mal ein netter Killer. In: Spiegel online, 04.04.2013: http://www.spiegel.de/kultur/tv/edelkrimi-serie-verbrechenim-zdf-nach-ferdinand-von-schirach-a-892075.html (27/01/2014).

Interview mit Ferdinand von Schirach im NDR: Sendung Das! vom 10.12.2013: http://www.ndr.de/fernsehen/sendungen/das/videos/dasx4213.html (27/01/2014).

KLUGE, Alexander / SCHIRACH, Ferdinand von. Der phantastische Einfallsreichtum der Realität. Ferdinand von Schirach im Interview: Dctp.TV. Online unter http://www.dctp.tv/\#/filme/ferdinand-von-schirach-verbrechen-aus-dem-alltag-einesstrafverteidigers/ (27/01/2014).

SCHECK, Denis. Unsichtbare Folterknechte. In: Der Tagesspiegel, 29.08.2010: http://www.tagesspiegel.de/kultur/aufgeschlagen-zugeschlagen-unsichtbarefolterknechte/1913428.html (27/01/2014).

SCHIRACH, Ferdinand von: Eine Geschichte hat immer ihre eigene Wahrheit. Rede zum Kleistpreis $2010 . \quad$ In: $\quad$ Der Tagesspiegel, 22.11.2010: http://www.tagesspiegel.de/kultur/rede-zum-kleistpreis-jeder-kann-zum-moerderwerden/3088620.html (27/01/2014).

SCHIRACH, Ferdinand von: Die Würde ist antastbar. In: Spiegel, 16.09.2013. http://www.spiegel.de/spiegel/print/d-112638562.html (27/01/2014).

recebido em: 28/02/2014 aceito em: 07/04/2014

Pandaemonium, São Paulo, v. 17, n. 23, Jun./2014, p. 1-25 